\title{
Discovering the Atomic Nucleus ${ }^{1}$
}

\section{Odilon A. P. Tavares}

Centro Brasileiro de Pesquisas Físicas - CBPF/MCT

22290-180 Rio de Janeiro - RJ, Brazil

E-mail: oaptavares@cbpf.br

XXXIV edition of the Brazilian Workshop on Nuclear Physics,

Foz de Iguaçu, Parana state, Brasil

5-10 June 2011

\footnotetext{
${ }^{1}$ In celebration of the centennial anniversary of the discovery of the atomic nucleus.
} 


\section{Prologue}

Nuclear physicists celebrate this year the centenary of the discovery of the atomic nucleus, announced in Manchester (England) in early 1911 by its discoverer sir Ernest Rutherford. This remarkable achievement not only represented the beginning of a new science, the Nuclear Physics, but was the basis and opened the way for understanding the real structure of atoms which make everything that is around us, including, of course, ourselves.

The present article has been thus prepared within the spirit of celebration of the centenary of the discovery of the atomic nucleus, which initiated a major scientific, philosophical, and technological revolution that has provided us with better days.

Humanity and, indeed, all life on our planet are supported at the expense of the energy that was and has primarily been generated, to a larger scale, in reactions between light atomic nuclei that are present in the Sun (thermonuclear fusion of hydrogen nuclei), and, to a much smaller scale, in the so-called nuclear fission (breaking of heavy nuclei such as uranium) which occurs in the nuclear reactors for electricity generation from nuclear power plants and also in the natural radioactivity exhibited by some unstable nuclei such as uranium, thorium, radium, samarium, and many others.

But how long ago and how did atomic nuclei become known? Who identified them as the ultra-minuscule central region of the atom which concentrates all the positive electric charge and virtually the entire atomic mass? One hundred years have passed since the public announcement by New Zealander physicist sir Ernest Rutherford (1871-1937), the discoverer of the atomic nucleus, in Manchester Literary and Philosophical Society on March 7, 1911.

Knowledge on atomic nuclei and the huge amount of technological applications resulting from that has made possible to modern man enjoy better health, comfort and wellbeing.

\section{Radiations and Radioactivity}

In the late nineteenth century, new and important discoveries in science, even taken as revolutionary, were made on entirely new phenomena that have caused to scientists of that time some perplexity, surprise, and even skepticism. In 1896 the French physicist Antoine Henri Becquerel (1852-1908) discovered a new type of radiation emitted by uranium salts that showed at least peculiar features. Such radiations, invisible, spontaneous, and of unknown origin, crossed bodies opaque to light, blackened photographic films, and were also found in thorium and its salts.

Investigations on these new radiations were taken again about two years later, especially by a young New Zealander, Ernest Rutherford, who was honored in 1895 with a studentresearcher scholarship, and, therefore, moved from Christchurch (New Zealand) to work at Cambridge University (England) under advice of the English physicist sir Joseph John Thomson (1856-1940). Also, in Paris, the French physicist Pierre Curie (1859-1906) and his wife, the Polish physicist and chemist Marie Sklodowska Curie (1867-1934), devoted themselves to the study of these radiations, leading them to discover two new chemical 
elements in 1898, which they named Radium and Polonium, both strong radiation emitters, for this reason called radioactive. In that same year, one of the first conclusions reached by Rutherford in Cambridge was that the radiations from uranium salts were of two very different types, both corpuscular in their nature, and he named them as alpha and beta rays. In Paris, Henri Becquerel, now full of enthusiasm about the news in the field of radioactivity, was able to demonstrate in 1900 that beta rays were identical, but having much more energy, to the corpuscles of cathode rays (particles with negative electric charge emitted from the cathode) of the experiments by sir JJ Thomson in 1897 . These led him to conclude that cathode rays were the fundamental particles that atoms were made of, calling them electrons.

By the beginning of last century it became evident to some physicists and chemists that atoms were the basic constituents of matter, and that they had in their structure electrons and positive charges, so as to ensure the neutrality of atoms. In addition, sir JJ Thomson was able to conclude from his experiments with cathode-ray tubes that the total mass of the electrons in any atom should be a very small fraction of the total atomic mass and, therefore, the mass of the atom should be associated to the positive electric charge.

In 1898, Rutherford moved to McGill University in Montreal (Canada), where he remained for a period of nine years. There he could enjoy better working conditions, and in 1899 Rutherford discovered a noble gas, radioactive, later named Radon. Rutherford together with the English chemist Frederick Soddy (1877-1956) discovered in 1902 the transmutation of radioactive elements, i.e. an element changes (or disintegrates, or decays) into another one due to spontaneous emission of alpha or beta rays [Fig. 1]. Rutherford concluded further that the alpha particles emitted from radon and radium had a positive electric charge because of the deflections that were observed when they passed in regions of intense electric fields.

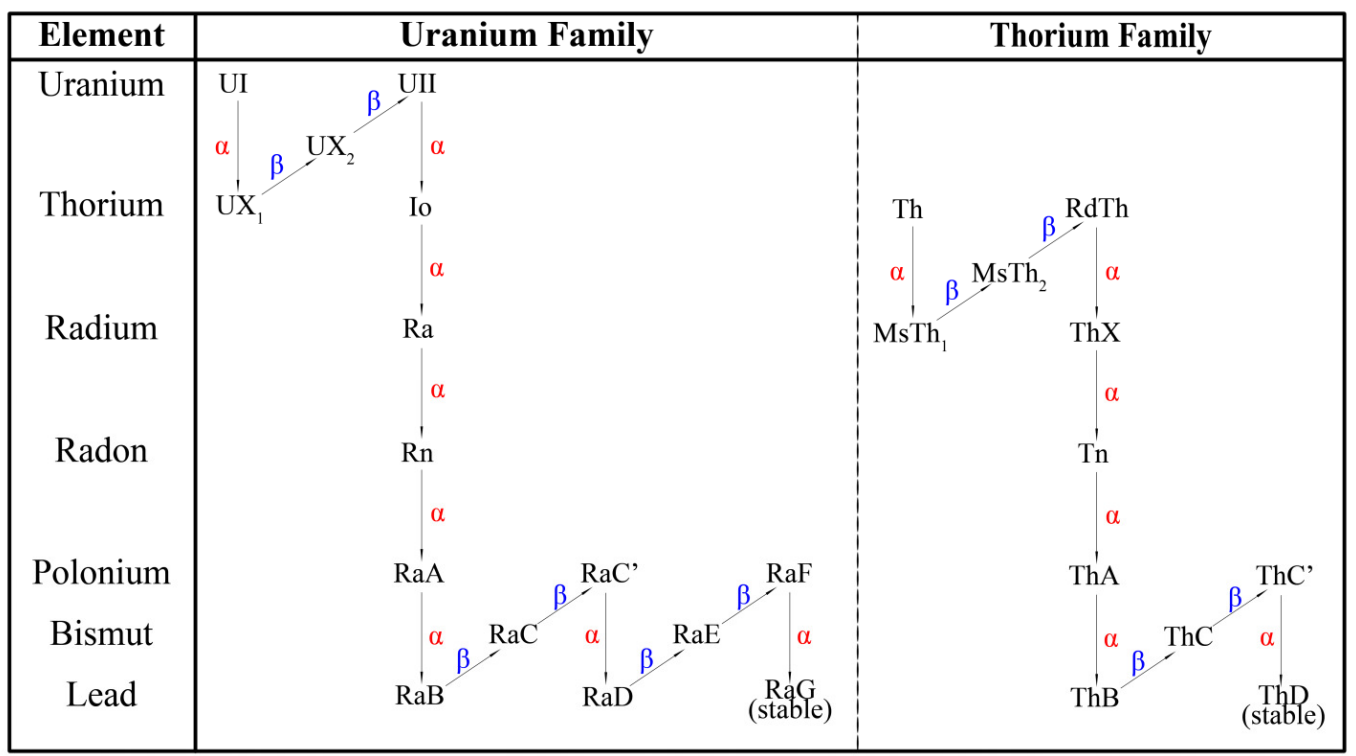

Fig. 1 By means of successive alpha (vertical arrows) and beta (inclined arrows) disintegrations, as time goes uranium and thorium transform into lead (stable element), after having generated radioactive elements in series (radium, radon, polonium, bismuth). 


\section{Ernest Rutherford and the alpha particles}

While in Montreal Rutherford had noticed that inside a vacuum chamber (box from where air had been removed) alpha rays were being slightly deflected from their initial direction when passing through very thin sheets of mica (about three hundredths of a millimeter) for a beam of them produced a diffuse spot on a photographic film.

Back to England in 1907, now at University of Manchester, Rutherford was able to gather around him brilliant and qualified young people who assisted and cooperated with him in a series of experiments that could confirm the alpha particles as being positively charged corpuscles.

A technique for counting alpha particles was developed by Rutherford and the German physicist Hans Geiger (1882-1945) when they knew in 1908 that it was possible to see light signals (scintillations) produced by alpha rays whenever they hit a screen coated with a layer of zinc sulfide (salt which exhibits the property of luminescence). This simple and successful method of counting alpha particles had become in the main tool of work in the experiments involving detection of alpha particles. The following year, with the help of another qualified assistant, the English physicist Thomas Royds (1884-1955), alpha rays were definitively identified as helium atoms that have lost both their electrons, therefore ions of double positive electric charge.

In Manchester, whenever Rutherford and his assistants used the zinc sulfide screen they observed inside a vacuum chamber that, when a beam of alpha rays passed through a small circular hole, the scintillations were observed in a well-defined circular area of diameter equal to that of the hole. However, when a thin sheet of mica was interposed between the hole and the screen, the region where the scintillation occurred became even greater, with edges not well defined, showing again that part of the alpha particles were deflected from its original direction [Fig 2].

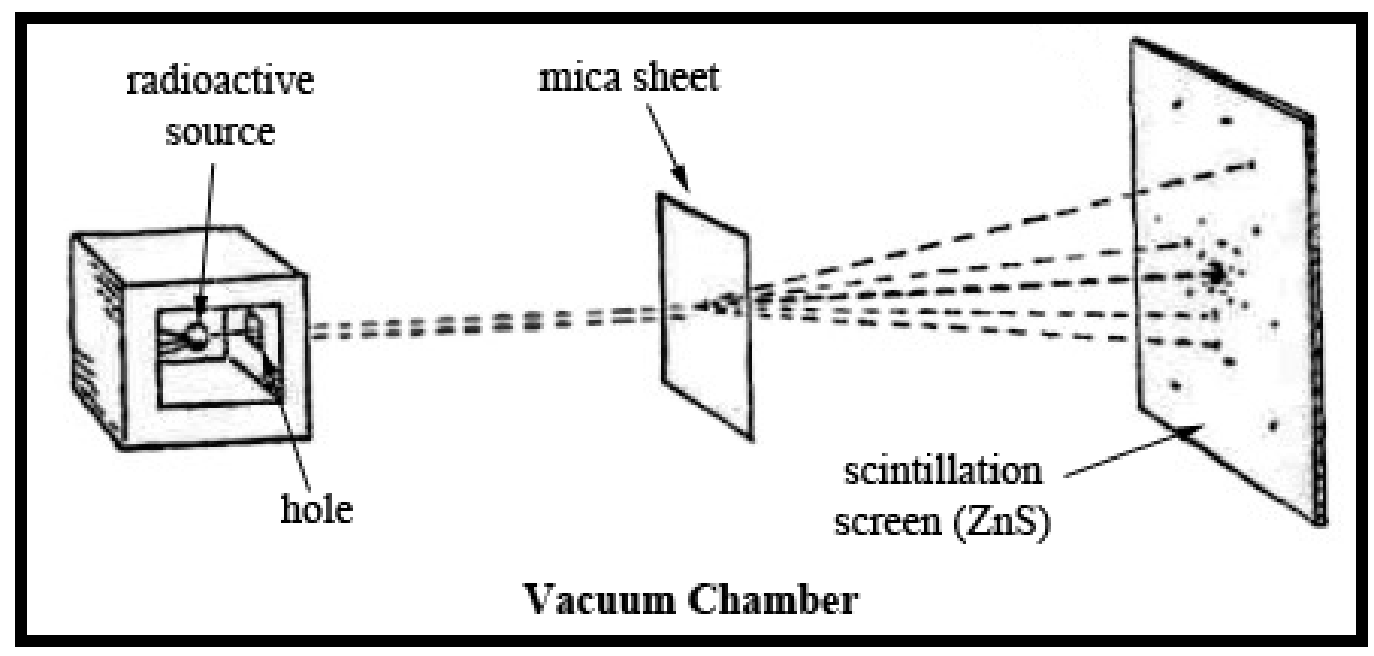

Fig. 2 Deflection of alpha particles by a thin mica sheet.

Without a convincing explanation for these puzzling observations, in 1909 Rutherford assigned a young student, the New Zealand-British physicist Ernest Marsden (1889-1970), the 
task of searching for alpha particles deflected at angles much greater than about $10^{\circ}$. Marsden, instead of using mica sheets, made use of very thin gold sheets, and to detect the alpha particles he used a zinc sulfide screen that could move in all positions, namely, in front of, to both sides, and even in the region near the incident beam, behind the gold target. With the aid of a small telescope focused on the screen it was possible to observe in a darkened room designed for that the individual points of light which indicated the number of alpha particles deflected at a given angle of scattering from the direction of incidence. To the astonishment of everybody, Marsden found that a few alpha particles were thrown backwards, hitting the scintillation screen when it was near the region of the incident beam, i.e., alpha particles were being deflected at angles much greater than $90^{\circ}$, i.e., they were actually being "reflected" by a very thin gold foil.

Rutherford and Geiger were able to demonstrate that if the current Thomson's atomic model (the one known as "plum pudding") was accepted as the correct model for the atom, the number of alpha particles deflected at angles greater than $\sim 10^{\circ}$ would become zero, which was in contradiction with observations. Thomson's model, which implied multiple scattering, could not explain conclusively the deviation of alpha particles to directions very different from the one of the incident beam, so little would be possible to explain those particles backscattered.

\section{Rutherford and the atomic nucleus}

At the end of 1910 Rutherford managed to glimpse the meaning of the backscattering of alpha particles when they struck on very thin gold sheets. And then he formulated his theory of alpha particle scattering according to which he considered essentially that the deflection was the result of a single interaction between the alpha particle (of double positive electric charge) and the positive charge of the atom, but now this latter being supposed to be concentrated in a tiny central region of the atom which he called atomic nucleus. The electrons, in turn, would be orbiting around the nucleus, like a planetary system, in a spherical region of radius comparable to the size of the atom (estimated at the time as $\sim 10^{-8} \mathrm{~cm}$ ). Rutherford also assumed that the atomic nucleus, considered the scattering center, would not experience any recoil because almost all the mass of the atom was to be concentrated there (for gold, the mass of the nucleus amounts to nearly fifty times that of the alpha particle). Finally, the repulsive force resulting from the positive electric charges of the alpha particle and the atomic nucleus, both considered in the model as point charges, followed the law of variation with the inverse square of the distance between the charge centers.

Rutherford's hypothesis of a scattering center, the nucleus of the atom, to explain deflections of alpha particles at large angles, also including backscattering, by means of a unique and rare impact between these particles and the atomic nucleus is entirely compatible with the observation that the great majority of incident alpha particles passed through the gold sheet without suffering appreciable deflections as if the sheet was transparent to them. In fact, for the gold leaf used by Rutherford's group it was possible to estimate as $\sim 6 \times 10^{-10} \mathrm{~cm}$ the average distance between the atomic nuclei as seen by the incident alpha particles. This meant that the chance for an alpha particle to pass near a nucleus (and thus suffer a deflection) was quite small, even rarer would be an incidence directly against the nucleus, with the resulting backscattering. Although small, these chances were, however, not null, in agreement with what was observed (recall that Marsden had recorded a single backscattering over 20,000 alphas!). The nearer the nucleus was to the direction of incidence the greater the deflection angle, and these events would be rarer until the extreme situation in which they practically kick in the original direction [Fig.3]. 


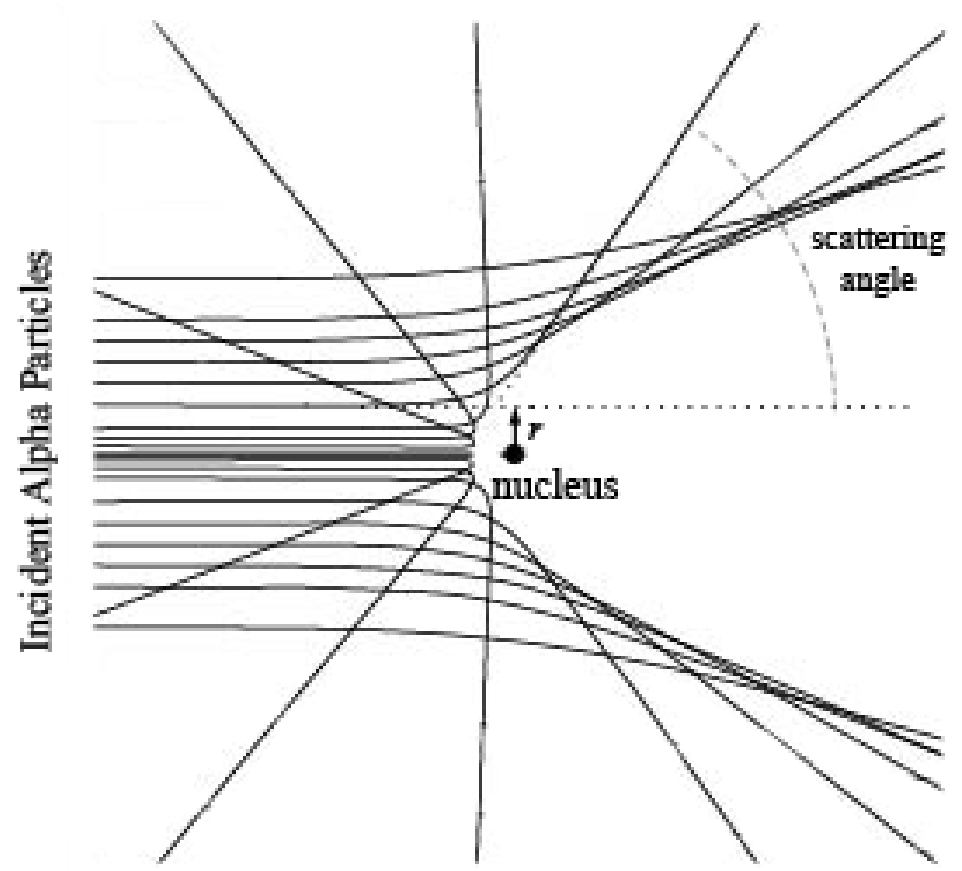

Fig. 3 The better the "aim" (low value of $r$ ) the rarer the large deflection angles (backscattering).

Presented to public for the first time on March 7, 1911, in Literary and Philosophical Society of Manchester, Rutherford's theory of nuclear atom to explain the scattering of alpha particles by thin metallic foils was published in detail in the following May in volume 21 of Philosophical Magazine. What made the nuclear model of the atom prevail over those of Thomson (1903) and Nagaoka (1904) was its ability to explain quantitatively the observations on the scattering of alpha particles by very thin metal foils. The conception of the nucleus of the atom, i.e. a very tiny central region containing the positive electric charge of the atom and practically (99.97\%) its entire mass held up itself for it was in complete agreement with the experiment. Afterwards, it was realized that the model contained serious limitations such as i) it failed to explain deflections at very small angles $\left(<2^{\circ}\right)$, ii) it did not take into account other forces, besides the electrostatic repulsion between the incident alpha particle and the target nucleus, which could eventually act at distances very close to the nucleus, and, especially iii) the inconsistency with atomic stability, as a result of the emission of radiation by the electrons in their movements around the nucleus, leading them to lose velocity gradually thus following a spiral path until finally falling in the nucleus, a fact that actually does not occur. These limitations, however, did in no way diminish the merits of the conclusion regarding the presence of a nucleus in the atom, an image which remains absolutely correct until present days. Rutherford, differently from Thomson and Nagaoka, realized that as a matter of fact the atom should be in fact a great empty space, and that its positive electric charge and mass should be concentrated in an ultra-minuscule central region which he named atomic nucleus (the matter density of the atomic nucleus could be estimated as great as 100 million tons per $\mathrm{cm}^{3}$ ).

During the years 1911-13 Geiger and Marsden undertook a series of experiments on scattering of alpha particles. They used thin metallic foils of silver, tin, copper, gold, and aluminum of different thicknesses, alpha particles of different velocities (from sources of radium and radon, and thin mica sheets as energy degrader), and scattering angles that covered the large interval $5^{\circ}-150^{\circ}$. In all cases Rutherford's theory of alpha particle scattering with the hypothesis of nuclear atom resulted confirmed within the experimental uncertainties. Only small 
deviations from the theory were noted in cases for aluminum targets or when using high speed alpha particles.

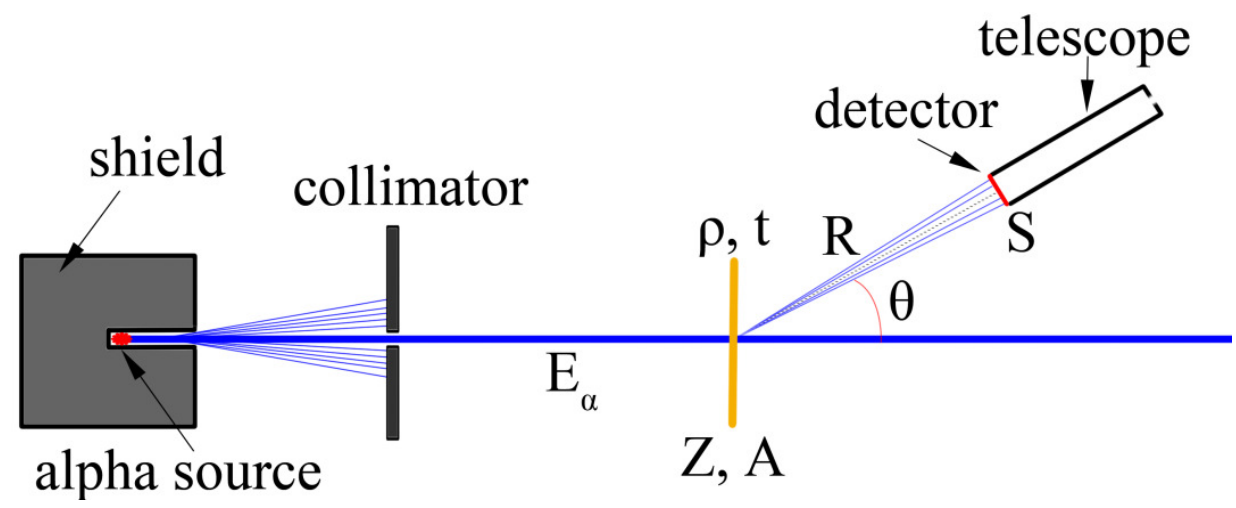

Fig. 4 Physical and geometrical quantities which enter into Rutherford's formula for the scattering of alpha particles by thin metallic targets

In the experiments to test Rutherford's model what was done was to count the number of alpha particles scattered at a given angle $\theta$ from the original direction of incidence by using a $\mathrm{ZnS}$ scintillation screen detector of known area $S$ placed at a distance $R$ from the scattering center [Fig.4]. If $Q$ represents the total number of alpha particles of kinetic energy $E$ which hits a thin metallic plate of thickness $t$, density $\rho$, atomic mass $A$, and nuclear charge Ze, Rutherford found for the number of alpha particles detected per unit area of the scintillation screen, $N / S$, the result

$$
N / S=C \cdot Q \cdot \frac{\rho}{A} \cdot \frac{t}{R^{2}} \cdot \frac{Z^{2}}{E_{\alpha}^{2}} \cdot \frac{1}{\operatorname{sen}^{4}(\theta / 2)}
$$

which is valid for scattering angles greater than about $1^{\circ}-2^{\circ}$, and where $\rho$ is expressed in $\mathrm{g} / \mathrm{cm}^{3}$, $t$ and $R$ in $\mathrm{cm}, S$ in $\mathrm{cm}^{2}, A$ in $\mathrm{g}, E_{\alpha}$ in $\mathrm{MeV}$ (million electron volt), and $C=3.12 \times 10^{-3} \mathrm{MeV}^{2} \cdot \mathrm{cm}^{2}$.

Rutherford's celebrated formula (1911) shows that the quantity $N / S$ results proportional to

i) the number of nuclei per unit volume (because this number is directly related to the ratio $\rho / A$ of the target material);

ii) the thickness of the metallic plate (provided that it should be very thin);

iii) the inverse square of the distance between the scattering center and the detector;

iv) the square of the electric charge of the nucleus;

v) the inverse square of the kinetic energy of the incident alpha particles;

vi) the inverse of the quantity $\sin ^{4}(\theta / 2)$. 


\section{Epilogue}

Having a character that impressed everyone, for his pioneering achievements in nuclear science, sir Ernest Rutherford - winner of the 1908 Nobel Prize in Chemistry, later Baron Rutherford of Nelson, and considered "scientist supreme" - is, according to the American astrophysicist Michael Hart, among the 60 most influential persons upon the destinies of humanity, and to the American science journalist John Simmons, among the 20 most important scientists of all fields of knowledge and of all time. Without any doubt, a beautiful path for a boy who was born in Spring Grove, in the rural province of Nelson (New Zealand), the son of a wheelwright and a schoolteacher, was buried beside the famous physicist sir Isaac Newton and the naturalist Charles Darwin in Westminster Abbey in London.

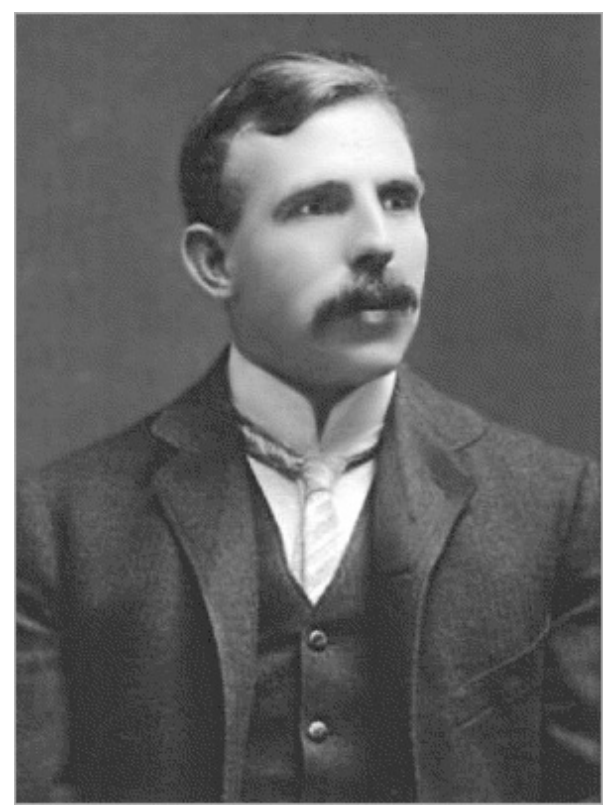

Ernest Rutherford (1871-1937), the discoverer of the atomic nucleus in 1911

\section{Bibliography}

[1] E. Rutherford, J. Chadwick, and C. D. Ellis, Radiations from Radioactive Substances, Cambridge University Press, Cambridge 1930.

[2] E. Segrè, From X-Rays to Quarks: Modern Physicists and their Discoveries, Dover Publications, 2007.

[3] Internet: www.nobel-winners.com/Chemistry/ernest_rutherford.html 